\title{
L'éducation des élèves roms en Slovaquie : entre ségrégation, assimilation, intégration et inclusion
}

The education of Roma pupils in Slovakia: Between segregation, assimilation, integration and inclusion

La educación de los alumnos romaníes en Eslovaquia : entre segregación, asimilación, integración e inclusión

René Lužica et Arnaud Segretain

\section{OpenEdition Journals}

Édition électronique

URL : https://journals.openedition.org/ries/6550

DOI : $10.4000 /$ ries.6550

ISSN : 2261-4265

Éditeur

France Education international

Édition imprimée

Date de publication : 1 septembre 2018

Pagination : 91-101

ISBN : 978-2-85420-619-7

ISSN : $1254-4590$

\section{Référence électronique}

René Lužica et Arnaud Segretain, « L'éducation des élèves roms en Slovaquie : entre ségrégation, assimilation, intégration et inclusion », Revue internationale d'éducation de Sèvres [En ligne], 78 | septembre 2018, mis en ligne le 01 septembre 2020, consulté le 25 juin 2021. URL : http:// journals.openedition.org/ries/6550; DOI : https://doi.org/10.4000/ries.6550 


\title{
L'éducation des élèves roms en Slovaquie : entre ségrégation, assimilation, intégration et inclusion
}

\author{
René Lužica \\ Université Constantin le Philosophe \\ de Nitra, Slovaquie \\ Arnaud Segretain \\ Institut national pédagogique \\ de la République slovaque
}

La démocratisation de la société tchécoslovaque, après 1989, a enclenché de nombreuses tendances positives, mais qui ont été marquées par des phénomènes négatifs. Le phénomène négatif le plus significatif est l'aggravation de la stratification sociale de la société. Une grande partie de la population vit dans la pauvreté et la privation socioculturelle. En effet, selon leur étude (2008) intitulée Les communautés roms dans l'est de la Slovaquie du point de vue de la recherche anthropologique de terrain, après avoir suivi de manière longitudinale la situation des villages roms en Slovaquie entre 1999 et 2005, Tomas Hirt et Marek Jakoubek notent que les Roms restent le groupe le plus problématique de la population slovaque. L'échec le plus cinglant a été vécu par la majorité des Roms vivant en communautés marginalisées dans des localités isolées en zones rurales et urbaines. Vivre dans un environnement isolé ne permet pas aux habitants de participer à la vie sociale commune; cela provoque de nombreux conflits avec le reste de la société et approfondit les stéréotypes et les préjugés qui font partie du discours public. Il est indiscutable que l'une des solutions clés dudit problème des Roms est l'éducation, et en particulier l'amélioration du niveau de celle-ci.

Un sociologue important avait écrit en 1961, déjà :

Le libéralisme et pseudo-humanisme jettent les Tsiganes dans l'isolement et font renaître le séparatisme indésirable des Tsiganes (...) Une tâche particulière est confiée à l'école, sa mission est de lutter pour l'enfant rom contre le retard de ses parents. Plus l'école pourra contrôler un nombre important d'enfants tsiganes, plus elle pourra influencer ces enfants et leurs parents (...) Les expériences pédagogiques montrent que les meilleurs résultats dans l'enseignement des enfants tsiganes sont atteints, lorsque tout effort et inclination séparatistes et isolationnistes sont supprimés. (Sus, 1961)

Ainsi, les racines de la situation postérieure à 1989 sont beaucoup plus profondes que l'on ne pourrait le penser, et sont repérables dès la fondation de 
la société socialiste. Cela signifie théoriquement que l'opinion que pouvait avoir la société (tchéco-) slovaque en 1961 a pu avoir une influence sur la situation post-1989. Ces dernières années, les thèmes de l'intégration puis de l'inclusion des élèves roms ont été régulièrement discutés. Nous comprenons l'intégration comme le processus d'acceptation d'un groupe minoritaire au sein d'un groupe majoritaire et l'inclusion comme un processus allant plus loin que l'intégration, à savoir la reconnaissance et l'appartenance à part entière de chaque individu, sans considération de ses différences, qu'elles soient d'ordre social, ethnique, culturel ou encore psychomoteur. L'environnement scolaire slovaque, cependant, ne réussit pas à respecter la situation spécifique et le contexte qui permettent de mettre les élèves roms sur le chemin de l'école. Mais ceux-ci ont aussi du mal à comprendre et à accepter les règles régissant les relations sociales et interpersonnelles à l'école, cette difficulté leur faisant in fine rejeter le système scolaire par peur et par incompréhension. Ainsi, l'intégration et l'inclusion demeurent des concepts théoriques que de nombreuses écoles expliquent sur la base d'opinions et de sentiments subjectifs.

Les racines de cette situation remontent à la période de l'établissement de la société socialiste, bien avant 1989. Alors qu'en 1992, le gouvernement de la République slovaque avait reconnu les droits de la minorité rom comme minorité nationale, le parti communiste au pouvoir ne reconnaissait pas l'existence d'une nation tsigane / rom en tant que minorité nationale. Les Tsiganes / Roms étaient considérés comme un groupe social arriéré. Quarante années d'efforts intenses pour rééduquer et assimiler la population rom ont laissé de profondes cicatrices sur la minorité rom et dans le processus éducatif. Nous nous attacherons dans un premier temps à rappeler les mesures qui ont été prises ces dix dernières années pour inclure à part entière les Roms dans le système scolaire slovaque et nous présenterons ensuite les résultats des dernières études visant à évaluer l'efficacité de ces mesures ayant eu pour objectif une meilleure intégration des Roms dans le système scolaire.

\section{MESURES PRISES DEPUIS 2008 POUR L'INCLUSION DES ROMS DANS LE SYSTÈME ÉDUCATIF}

Le système éducatif a un rôle clé à jouer dans le renforcement de l'homogénéité des droits et des devoirs de chacun dans la société. Les enseignants communiquent à chaque génération les valeurs de la majorité commune de la société, et les inculquent aussi aux minorités. Le système éducatif fait partie du monopole du pouvoir de l'État. Dans le passé, jusqu'au milieu du XX $\mathrm{X}^{\mathrm{e}}$ siècle, l'État était intolérant, assimilationniste, ségrégationniste envers les minorités. Depuis les années soixante du siècle dernier, il est progressivement devenu interculturel, biculturel, multiculturel, inclusif. En Tchécoslovaquie, ou plutôt en Slovaquie, ces processus ont commencé à être mis en œuvre seulement à la 
fin des années 1990. En dépit des développements positifs au cours des vingt dernières années, on a constaté que les élèves roms n'apprenaient pas dans leur langue maternelle et n'apprenaient rien de leur propre histoire ni de leur culture traditionnelle et contemporaine. Ainsi, leurs condisciples non roms ne s'enrichissaient pas non plus.

Des matières telles que la langue rom, l'histoire des Roms, la culture rom ont alors été incluses dans les programmes de formation continue du personnel enseignant des écoles primaires, des assistants et des éducateurs des clubs scolaires. Les écoles peuvent intégrer ces " matières roms » et choisir de les mettre en œuvre dans le cadre de l'enseignement de l'histoire, de l'éducation civique, dans le cadre quotidien du projet pédagogique de l'école ou encore lors d'activités de loisirs. L'école, la classe ou le club inclusif devraient considérer la langue rom sous son aspect positif, offrir suffisamment d'espace pour développer les compétences de communication des élèves roms, offrir un espace pour la présentation de la vie linguistique et culturelle des élèves roms et de leurs parents, créer et utiliser des matériaux de l'histoire locale, régionale des Roms ; les professionnels de l'éducation devraient recevoir des commentaires des élèves et de leurs parents et organiser régulièrement des rencontres avec ces derniers en programmant des activités linguistiques et culturelles. De nombreuses études montrent que la création d'un environnement scolaire inclusif est un processus difficile, long et souvent pétri de contradictions.

Dans le cadre de l'éducation et de la formation des minorités nationales, le gouvernement de la République slovaque a adopté, en 2007, une Conception pour l'éducation et la formation des minorités nationales, puis en 2008, une Conception pour l'éducation et la formation des enfants et des élèves roms, y compris pour le développement de l'enseignement secondaire et supérieur. Dans ces deux documents, ainsi que dans la Stratégie de la République slovaque pour l'intégration des Roms jusqu'en 2020, adoptée en 2012, l'accent est mis sur l'éducation préscolaire des enfants à partir de trois ans et sur l'éducation des enfants des communautés roms marginalisées, afin d'améliorer leur préparation à l'entrée en première classe de primaire, de réduire le nombre d'enfants fréquentant une école primaire spécialisée et d'utiliser la langue maternelle, lorsque les enseignants travaillent avec des élèves roms. Déjà, en 2004, le ministère de l'éducation nationale slovaque avait adopté des Mesures temporaires d'équilibrage pour préparer un nombre suffisant de pédagogues enseignant en langue rom. En 2011, l'Institut national pédagogique a officialisé un programme d'enseignement pour la langue et la littérature roms (parmi les autres matières du pilier Langue et communication). Ses standards de contenu et de performance sont axés sur la langue, la littérature, l'histoire et la culture roms. En première et deuxième année, les élèves acquièrent des informations de base et en troisième et quatrième année, ces connaissances sont élargies. Lors de la même année (2011), l’Institut national pédagogique a validé la poursuite de ce programme d'enseignement au collège et a fixé les exigences du cadre des compétences linguistiques à atteindre par les 
élèves au niveau A2. L'enseignement de cette matière est possible de la cinquième à la neuvième année de l'école primaire (collège slovaque) et de la première à la cinquième année du cursus du lycée, qui dure huit ans (collège et lycée regroupés en un seul établissement en Slovaquie). Le ministère de l'éducation nationale a validé en 2013 le cadre des connaissances et compétences exigées pour passer le baccalauréat en langue et littérature roms, et qui sont en parfaite conformité avec les standards de contenu et de performance validés dans le programme d'enseignement de la langue et la littérature roms pour les établissements secondaires (lycées slovaques) ainsi qu'avec les documents internationaux du Conseil de l'Europe pour enseigner le romani.

Selon Milan Samko, spécialiste de la langue et la culture roms :

ces mesures sont efficaces, mais nous n'avons pas encore été en mesure de préparer un nombre suffisant d'enseignants de langue et culture roms. Aujourd'hui, par exemple, il est temps de rouvrir les Mesures temporaires d'équilibrage et d'envisager de les mettre à jour (Samko, 2014)

C'est un paradoxe historique qu'en 1953, le Conseil scientifique de l'Institut oriental de l'Académie des sciences de Tchécoslovaquie ait nommé un comité pour l'étude de la langue et la culture roms, qui avait établi une base théorique pour des approches de travail avec la minorité rom. Le résultat de ce travail d'universitaires fut la publication, en 1974, du manuel Notions de romani. La direction du Parti communiste refusa cependant de publier ce manuel et les activités de ces universitaires furent considérées comme réactionnaires (Nečas, 2008). Contrairement au passé, aujourd'hui, les manuels de langue et culture roms sont imprimés. Mais d'un autre côté, ils ne sont pas inclus dans le processus d'enseignement, parce qu'il y a un manque flagrant d'enseignants compétents pour enseigner ces matières. D'ailleurs, les élèves roms obtiennent continuellement les moins bons résultats dans les tests scolaires. Et les enseignants n'ont pas l'obligation d'enseigner en particulier à des élèves roms. Ils enseignent à tous les élèves, sans distinction ethnique ou d'origine, dans toutes les écoles. La discrimination, même si celle-ci peut être positive, des enseignants selon leur origine rom ou autre, pour répondre à des besoins spécifiques, quels qu'ils soient, n'existe pas. On peut ajouter aussi, à ce titre, ce que mentionne le recensement de l'année 2001 en Slovaquie, où, selon les estimations du Bureau des statistiques de la République slovaque, trois quarts des personnes d'origine rom n'auraient pas déclaré leur origine rom et se seraient même déclarées d'origine slovaque ou magyare.

\section{ÉTUDES RÉCENTES \\ SUR L'IMPLÉMENTATION \\ DE CES MESURES}

La plupart des études sont axées sur l'efficacité de l'implémentation de ces mesures à un niveau macro. Les niveaux méso et micro ont été considérés 
comme non pertinents et trop subjectifs. L'institution dominante est l'institution d'État - le ministère de l'éducation nationale qui assure la mise en œuvre de la politique éducative. Les résultats des études quantitatives permettent d'identifier les causes et les méthodes d'exclusion, de ségrégation des élèves issus de communautés roms marginalisées, leur fonctionnement et la manière dont elles apparaissent dans la pratique scolaire. Ces dernières années, les méthodes qualitatives ont été préférées pour mettre en évidence les implications de la politique éducative, laquelle produit (mais pas nécessairement intentionnellement) des pratiques de ségrégation touchant les acteurs eux-mêmes : les élèves roms, les enseignants, le personnel éducatif, les parents.

\section{L'Étude sur les Parcours ÉDUCATIFS DES ÉLĖVES ROMS ISSUS DE MILIEUX SOCIALEMENT DÉFAVORISÉS}

Parmi les principales activités de recherche, on trouve l'étude sur les Parcours éducatifs des élèves roms issus de milieux socialement défavorisés des chercheurs Alice Petrasová et Štefan Porubský (2013), qui n’a pas été réalisée à la demande du ministère de l'éducation mais dans le cadre du secteur non gouvernemental (Open Society Foundation). Les conclusions montrent que la moitié des élèves roms considèrent que leurs enseignants sont bons, l'autre moitié qu'ils sont mauvais. La plupart d'entre eux ont estimé qu'ils n'étaient pas eux-mêmes de très bons élèves. Les principales causes de cet état de fait sont une motivation insuffisante, un manque de modèles et un faible soutien de la part de la famille. La plupart des élèves roms ont exprimé des sentiments négatifs à propos de leurs relations avec leurs camarades de classe non roms. Ils ont justifié cela par des obstacles à la communication ainsi qu'un comportement méprisant. Un bon enseignant est, selon cette étude, celui qui ne crie pas et qui est amical envers tous, sans distinction. La plupart des élèves roms aspirent à un bon travail et à une bonne famille. Certains ont indiqué que leur origine ethnique était un obstacle pour accéder au marché du travail. Un faible nombre d'élèves aimerait vivre sans travailler. Les élèves roms ont exprimé une préférence envers des matières comme les mathématiques, l'éducation physique et sportive et le slovaque. Seul un nombre minime d'entre eux est satisfait de sa vie actuelle.

\section{Le PROJET PRINED (2015)}

Le projet PRINED - PRojet d'ÉDucation INclusive (2015) est le produit du Centre méthodo-pédagogique, qui est l'institution pédagogique du ministère de l'éducation nationale slovaque en charge de la formation continue des professeurs. Ce rapport s'efforce de défendre l'activité de cette institution, qui assure la formation des enseignants et du personnel éducatif par divers programmes inclusifs. L’objectif principal des projets nationaux déjà achevés en 2015 était de 
créer un environnement inclusif pour cent écoles primaires et cinquante écoles maternelles. Il s'agissait d'améliorer les compétences professionnelles de centaines de personnels éducatifs et spécialisés. Dans les écoles maternelles, le but était d'améliorer le processus de diagnostic et de créer des équipes composées d'enseignants et de spécialistes dans les écoles primaires. Les chercheurs se sont concentrés sur la compréhension du modèle de l'école inclusive. Les résultats ont montré que la plupart des employés des écoles primaires considéraient leur école comme inclusive. Ils estimaient de façon positive les progrès des élèves, l'amélioration et l'extension des services, l'amélioration de l'atmosphère dans l'école et la coopération avec les parents (Rapport d'évaluation, 2015a). La même année a été publié le rapport d'évaluation du projet PRINED pour un modèle inclusif d'éducation dans les écoles maternelles. Les chercheurs y abordent en particulier les faiblesses de l'éducation préscolaire pour les enfants roms. Ils y identifient une faible sensibilisation des parents vis-à-vis des avantages de l'éducation préscolaire, un niveau élevé de méfiance de la part des parents envers l'institution scolaire, des barrières linguistiques (les enfants roms communiquant en romani), une fréquentation insuffisante et irrégulière de la maternelle, des attitudes négatives de la part du reste de la société majoritaire, des capacités insuffisantes, un manque d'assistants parlant la langue rom, de faibles compétences professionnelles du personnel enseignant pour travailler avec les enfants roms dans des localités d'exclusion sociale et une mauvaise coopération entre les écoles maternelles et les autres participants potentiels à l'éducation des enfants roms (centres communautaires, travailleurs sociaux sur le terrain, etc.). Selon les enseignants, le modèle éducatif inclusif est un long processus, dont la pierre angulaire est la sensibilisation des parents, des enseignants, la fréquentation de l'école de la part des enfants à partir de 4 ans au moins. Les enfants ont un temps d'adaptation plus long à l'environnement et, s'ils entraient dans un modèle inclusif à 5 ou 6 ans, ils seraient moins mesure de s'adapter aux autres enfants et de participer au processus pédagoéducatif (Rapport d'évaluation sur la mise en ouvre du modèle inclusif, 2015b).

\section{L'ANALYSE MENÉE PAR L'INSTITUT NATIONAL PÉDAGOGIQUE (2016-2017)}

Entre 2016 et 2017, l'Institut national pédagogique a réalisé pour le ministère de l'éducation nationale slovaque une Analyse de l'état actuel des processus d'intégration et d'inclusion dans les conditions des écoles slovaques. Cette étude complète a inclus la formation des élèves ayant des besoins éducatifs spécifiques. Elle a dressé une carte des niveaux d'exclusion de l'environnement scolaire entre le $1^{\text {er }}$ août 2016 et le 15 avril 2017. L'étude a interrogé la direction de l'école, le personnel pédagogique, le personnel spécialisé, les parents. L'échantillon choisi représentait proportionnellement toutes les régions, tous les types d'écoles (publiques, privées, confessionnelles), les écoles dans les villes et les 
zones rurales. Les écoles ont été sélectionnées de façon que soient représentés trois groupes d'élèves : les handicapés, ceux issus de milieux socialement défavorisés et ceux intellectuellement précoces. Le nombre minimum d'élèves inclus

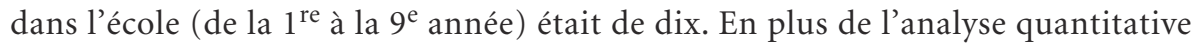
(avec un questionnaire comme outil de recherche), l'analyse qualitative orientée (avec un entretien comme outil de recherche) a été utilisée. ${ }^{1}$

Sur l'échantillon choisi pour l'étude quantitative, 737 questionnaires sur la question de l'inclusion dans l'environnement scolaire sont revenus de la part de 134 écoles représentatives (taux de retour : 53,6 \%). Les indicateurs évalués sur une échelle de 1 à 5 ont été les suivants :

(1) conditions générales pour les activités scolaires et l'utilisation des ressources ;

(2) spécificités du processus éducatif ;

(3) législation, gestion et organisation;

(4) interaction des professionnels, des élèves et des parents ;

(5) postures vis-à-vis de l'éducation inclusive ;

(6) coopération avec d'autres établissements ;

(7) formation initiale et continue du personnel pédagogique et spécialisé.

Les scores les moins bons ont été respectivement relevés pour les indicateurs (6), (1) et (7). L'indicateur (3) est relativement bien évalué, avec par exemple $82,3 \%$ des répondants déclarant que les horaires et programmes d'enseignement de leur école reflètent bien le niveau et le rythme d'apprentissage de chacun des élèves. Cette relative bonne impression de la législation reflète les efforts institutionnels mentionnés plus haut, lesquels ont été menés pour concevoir un programme d'enseignement pour tous les niveaux d'enseignement, jusqu'à la définition d'un cadre de connaissances et compétences à atteindre pour passer le baccalauréat en langue et littérature roms.

Le corpus de recherche pour l'étude qualitative comprenait des écoles élémentaires publiques, privées et confessionnelles, de dix municipalités. Les écoles ont été sélectionnées selon leur région d'appartenance : trois en Slovaquie occidentale, trois en Slovaquie centrale et quatre en Slovaquie orientale. Les critères de sélection des répondants de chaque école étaient les suivants : la direction de l'école ou la direction adjointe (1), le professeur d'école primaire (1), le professeur principal au collège ( $2^{\mathrm{e}}$ niveau de l'école primaire slovaque) (1), le personnel spécialisé (2) comme le psychologue scolaire, le pédagogue spécialisé, etc., et le représentant des parents d'élèves (1). Le nombre d'entretiens a été de 60 .

La problématique de cette étude qualitative peut être caractérisée par la question suivante : "Quelles sont actuellement les conditions et les obstacles à

1. Le garant de la partie quantitative de l'étude était le professeur Viktor Lechta, celui de la partie qualitative était le professeur René Lužica et le coordinateur des travaux était le docteur Jozef Facuna. 
l'éducation inclusive dans les écoles primaires en Slovaquie, afin de proposer des solutions et d'identifier des priorités pour créer les conditions de l'éducation inclusive?»

Sur la base de l'étude qualitative des échantillons des écoles étudiées, nous pouvons constater une diversité des pratiques professionnelles pour la promotion et la mise en œuvre de l'éducation inclusive. Cette variabilité constatée est causée par les différents points de vue et attitudes des directions, des enseignants et des éducateurs spécialisés. Un rôle négatif est joué par le stéréotype assimilationniste et monoculturel toujours présent du "modèle éducatif socialiste », auquel les plus âgés des enseignants interrogés étaient habitués. Cet état de fait aggrave encore la discrimination et la stigmatisation des enfants roms et de leurs parents, qui sont convaincus que la société majoritaire ne les respecte pas et qu'ils ne méritent pas le respect et la dignité accordée à tous les autres. Standardiser les contenus, les formes, les méthodes et motiver les acteurs mettant en œuvre l'éducation inclusive est la tâche du ministère de l'éducation nationale slovaque, des académies et des autorités locales et régionales tutélaires, afin d'assurer la formation continue, en particulier du personnel enseignant et des assistants. Cette tâche est aussi celle des facultés de pédagogie, qui devraient pouvoir répondre de façon plus flexible à la demande de préparation des futurs enseignants à l'inclusion dans le processus d'enseignement des élèves handicapés ou socialement défavorisés, mais aussi des élèves intellectuellement précoces. Le groupe spécifique le plus important est celui des élèves roms qui, dans de nombreux cas, combine plusieurs désavantages. Les élèves roms ont un problème psychologique commun de réalisation de soi non satisfaite et non soutenue en raison de leur "différence ". Si l'éducation est la clé du succès dans la vie, les facultés de pédagogie devraient créer des programmes d'études qui mettent l'accent sur l'étude de l'altérité, les schémas d'acceptation, de connaissances socio-psychologiques, ethnoculturelles et socio-anthropologiques qui soutiennent une stratégie de changement de paradigme, à savoir le fait de s'affranchir des préjugés et de la vision pleine de contradictions de la majorité sur la minorité. Cette étude montre que l'inclusion bute sur un mécanisme systémique insuffisant de soutien spécialisé et financier. Le ministère de l'éducation n'a pas de fonds de soutien financier pour l'inclusion. Les activités éducatives à court terme des organisations gouvernementales et non gouvernementales, les études partielles n'influencent qu'en partie la pratique et ne conduisent pas à un changement qualitatif au niveau national. La véritable mise en œuvre de l'éducation inclusive en Slovaquie est un processus à mener sur le long terme et dont l'application nécessite des efforts professionnels concertés, avec le soutien financier maximal et continu de l'État.

L'école reste pour les Roms un environnement étranger, malveillant et dans une langue étrangère. Il existe plusieurs explications historiques, et nombre d'entre elles sont déjà devenues " populaires » et partie intégrante du discours public. Les Roms sont à l'origine une culture nomade, dont une caractéristique 
dominante est l'analphabétisme. Dans leur patrie d'origine, en Inde, les Roms appartenaient à de basses castes, auxquelles l'apprentissage était interdit. Les Roms n'ont pas créé d'État et, dans chaque pays, ils se retrouvent au sein d'une minorité discriminée. Ils n’ont pas créé de nation basée sur une langue commune, un territoire, des expressions culturelles, des fondements psychologiques, une histoire commune et une coopération économique. Les Roms ne connaissent pas le concept de nationalisme, qui mène inévitablement à une conscience nationale d'appartenance à une population composée d'habitants dirigeants et dirigés.

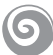

Ces dernières années, tous les documents se déclarent favorables à la politique d'inclusion mais, dans la réalité scolaire, l'inclusion bute sur un certain nombre d'obstacles objectifs et subjectifs. Force est de constater que restent toujours valables aujourd'hui les conclusions du rapport de Petrášová et Porubský (2013), dans lequel les auteurs ont identifié les principaux obstacles à la mise en œuvre réussie des principes de l'inclusion scolaire des élèves roms :

- une définition peu claire des catégories ciblées par la politique éducative de l'État dans ce domaine et une incohérence des objectifs partiels et des stratégies ;

- un manque de "volonté politique » de traiter ce phénomène comme l'une des priorités de la politique gouvernementale (et non comme une question concernant seulement le ministère) et de créer les conditions pour harmoniser le cadre législatif et les ressources budgétaires nécessaires à leur mise en œuvre ;

- une non prise en compte des conditions historiques, régionales et socioculturelles dans lesquelles le problème de l'exclusion des communautés roms s'est développé ;

- une perception des réformes scolaires qui se situe essentiellement à un niveau organisationnel (comment le système est organisé) et seulement dans une faible mesure à un niveau institutionnel (comment le système fonctionne), ce qui se manifeste principalement par la rigidité du système éducatif mis en œuvre dans le processus d'apprentissage en classe - la réforme se terminant donc à la porte des salles de classe.

En termes de parcours éducatifs et de perspectives des élèves roms issus de milieux socialement défavorisés, les auteurs (2013) mettent en évidence les facteurs déterminants suivants :

- un confinement du système et une faible sensibilité interculturelle du système éducatif scolaire ;

- une mentalité professionnelle des enseignants et du personnel spécialisé qui ne permet pas leur adaptation au modèle inclusif;

- une marginalisation non seulement sociale mais aussi culturelle toujours plus importante et un isolement d'une partie non négligeable des communautés roms; 
- une impuissance sociale et culturelle des élèves roms issus de milieux socialement défavorisés, incités à quitter leur communauté sans véritable soutien.

Selon les personnes interrogées dans le cadre de cette étude, les causes de cet échec et des problèmes dans l'éducation des élèves roms sont les Roms eux-mêmes, leur altérité culturelle, leur mode de vie, leurs choix de vie. Ni les directions d'écoles ni les enseignants ni le personnel spécialisé n'ont identifié la cause des difficultés concernant l'éducation des élèves roms comme provenant du système éducatif scolaire ou de l'école. La mentalité professionnelle des enseignants et du personnel spécialisé n'est pas habituée à respecter l'interaction interpersonnelle et interculturelle parmi les élèves, les activités de coopération entre les élèves étant presque totalement absentes du processus d'apprentissage. Il n'y a pas de place pour le dialogue, la discussion, l'argumentation. Il est presque symptomatique que seul quelques enseignants encouragent et soutiennent les relations positives entre élèves roms et non roms.

De façon localisée, le système éducatif slovaque est confronté à la réalité de certaines écoles, où la majorité des élèves roms sont aussi physiquement handicapés et/ou issus de milieux socialement défavorisés et où sont minoritaires les élèves roms sans handicap, parmi lesquels se trouvent parfois des élèves intellectuellement précoces. Les élèves non roms ont déménagé de ces localités ou fréquentent l'école primaire la plus proche, où se trouvent seulement des élèves non roms. Ainsi, l'application de l'inclusion scolaire a conduit à une forme de ségrégation scolaire.

Les efforts entrepris au niveau institutionnel ne se reflètent donc pas encore suffisamment dans la pratique éducative des enseignants dans les écoles. Il est à ce titre impossible de ne pas mentionner les propos de la Défenseuse des droits slovaque, Maria Patakyova, qui, selon une étude réalisée par son Bureau en mai 2018, alertent le gouvernement sur la ségrégation persistante dans les écoles slovaques, alors que les textes législatifs slovaques l'interdisent formellement. Selon Patakyova (2018), l'une des raisons est que malgré leur interdiction formelle, ces pratiques ne sont en réalité pas sanctionnées. Depuis 2014, la Commission européenne critique cette ségrégation dans les écoles slovaques et Maria Patakyova prévient que si la situation ne change pas, la Slovaquie risque d'être sujette à une procédure d'infraction pour discrimination et ségrégation systématiques des enfants roms dans le système scolaire.

\section{BibLIOgRAPHIE}

Collectif d'auteurs (2008): Rómske osady na východnom Slovensku z hladiska terénneho antropologického výskumu. Zborník vybraných výsledkov projektu Monitoring situácie rómskych osád na Slovensku 1999-2005. Bratislava : Open society Foundation, ISBN 978-80-969271-5-9.

Collectif d'auteurs (2015a) : Evalvačná správa z projektu PRINED - Projekt INkluzívnej Edukácie, Bratislava : Centre méthodo-pédagogique, ISBN 978-80-565-1415-3. 
Collectif d'auteurs (2015b) : Evalvačná správa z implementácie inkluzívneho modelu vzdelávania $v$ materských školách II, Bratislava: Centre méthodo-pédagogique, ISBN 978-80-565-1416-0.

Collectif d'auteurs (2017): Analýza aktuálneho stavu procesov integrácie a inklúzie $v$ podmienkach slovenského školstva, Bratislava: Institut national pédagogique pour le Ministère de l'éducation, de la science, de la recherche et du sport de la République slovaque.

NECAS C. (2008) : Historický kalendár. Déjiny českých Romú v datech, Olomouc: Université Palacky, ISBN 978-80-244-1747-9.

PATAKYOVÁ M. (2018) : Správa verejnej ochrankyne práv o stave prijatia navrhovaných opatrení z rokov 2013, 2014 a 2015 v oblasti výchovno-vzdelávacieho procesu na Slovensku s cielom zlepšovania ochrany a dodržiavania základných práv a slobôd osôb, Bratislava: Bureau du Défenseur public des droits. En ligne [https://bit. ly/2Mpn4Jr]

PETRASOVÁ A., PORUBSKÝ Š. (2013) : Vzdelávacie cesty rómskych žiakov zo sociálne znevýhodneného prostredia. Správa z výskumu, Bratislava: Nadácia otvorenej spoločnosti - Open society Foundation, ISBN 978-80-89571-08-6.

SAMKO M. (2014) : Inkluzívny vzdelávací program: jazyk a kultúra žiakov z MRK $v$ inkluzívnom vzdelávaní, in collectif d'auteurs (2014), Pedagogický model inkluzívneho vzdelávania v základných školách. PRINED - Projekt inkluzívnej edukácie, Prešov: Centre méthodo-pédagogique. ISBN 978-80-565-0208-2.

SUS J. (1961) : Cikánská otázka v ČSSR, Prague: Státní nakladatelství politické literatury. 
